\title{
Age-related diseases: common or diverse pathways?
}

\author{
Lloyd Demetrius • Vadim E. Fraifeld
}

Published online: 4 December 2014

(C) Springer Science+Business Media Dordrecht 2014

First of all, we dedicate this special issue of Biogerontology to Professor Brian F. C. Clark, Ph.D., Sc.D., in honor and memory of his tremendous support and promotion of aging research in Europe and across the globe. Brian Clark was instrumental in the establishment of several initiatives in Denmark and other countries towards basic biogerontology and translational research across aging and age-related diseases.

The recognition that aging is a major risk factor for the late-onset forms of cancer, neurodegenerative disorders, diabetes, and other age-related diseases (ARDs) has stimulated an extensive search for a common molecular basis underlying the origin and propagation of these pathologies. Along with the discovery of several common processes, such as metabolic reprogramming and cellular senescence, emerging evidence indicates that these processes could also diverge at some point, leading to one pathology or another. An important example of

\section{Demetrius $(\bowtie)$}

Department of Organismic and Evolutionary Biology,

Harvard University, Cambridge, MA 02135, USA

e-mail: ldemetr@oeb.harvard.edu

\section{E. Fraifeld $(\bowtie)$}

The Shraga Segal Department of Microbiology, Immunology and Genetics, Center for Multidisciplinary Research on Aging, Ben-Gurion University of the Negev, POB 653, 84105 Beer Sheva, Israel

e-mail: vadim.fraifeld@gmail.com convergence and commonality is an inverse association of cancer with age-related neurodegenerative disorders. This inverse co-morbidity suggests a metabolic basis for the late-onset forms of cancer and neurogenerative disorders, such as Alzheimer's disease, Parkinson's disease and amyotrophic lateral sclerosis.

The authors of this special issue have reviewed different aspects of the links between ARDs, and between ARDs and basic mechanisms of aging, from the genetic ("nuclear-genomic") and/or metabolic ("bioenergetic") perspectives. Jane Driver, one of the pioneers in the statistical analysis of epidemiological data on ARDs, gives a concise evaluation of the biological and epidemiological evidence (Driver 2014). As stressed in the paper, an investigation of the relation between cancer and neurodegenerative disorders requires a careful analysis of the non-biological factors which may corrupt the data and disturb the analysis. Such an analysis requires a sophisticated statistical methodology which can distinguish between biological and the non-biological factors. Driver's review achieves this distinction. The analysis thus succeeds in evaluating the significance of the relation between late-onset forms of cancer and Alzheimer's or Parkinson's diseases, but also between cancer and the less-common neurodegenerative disorders such as Huntington's diseases, schizophrenia and multiple sclerosis-disorders where non-biological 
factors are known to have a critical influence on the incidence and progression of the pathology. The problem of elucidating the molecular mechanisms that underlie the relations between ARDs is of a different character. The mechanisms fall roughly into two broad categories. The first lies within the nuclear-genomic perspective, while the second is more related to the bioenergetic model. The factors implicated include shared genes and pathways which are dysregulated in different directions to cause one disease or the other. Campus-Melo et al. (2014) have focused on RNA-binding proteins (RBPs) as links between cancer and neurodegenerative disorders. RBPs have a broad range of functions including mRNA export and localization, and post transcriptional regulation of mRNA stability. Accordingly, they will be integral elements in any study of the relation between cancer and neurodegenerative disorders. The pertinence of RBPs as molecular links between the two classes of disorders is delineated by the studies which show that alterations in these key proteins of RNA metabolism could impact the mechanisms of cell cycle, cell survival and cell death, leading to cancer or neurodegeneration. In view of their broad activities, RBPs may impact the energy metabolism as well.

The significance of the bioenergetic viewpoint is illustrated by the articles by Fu and Jhamandas (2014) and Harris et al. (2014). The nuclear-genomic models of $\mathrm{AD}$ and other neurodegenerative disorders have generally excluded the impact of astrocycles on the origin and propagation of the diseases. These cells, as emphasized in the astrocyte-neuron lactate shuttle model of Pellerin and Magistretti (2012), provide substrates for neuronal activity and play a critical role in the transition towards pathogenesis. Fu and Jhamandas gives a review of how defects in astrocytic metabolism may affect the astrocytic-neuronal relations and drive the system towards diseased states. Harris et al. (2014) extensively review the biochemical mechanisms that underlie the relation between cancer and AD. The authors deal both with particular genes, such as PinI, which may influence both cancer and $\mathrm{AD}$, and the metabolic pathways which are common to the diseases. This review also describes the effect of metabolic interventions as a therapy. The article by Demongeot and Taramasio (2014) is concerned with obesity, type 2 diabetes and neurodegenerative disorders. This paper discusses the effect of social networks on the spread of obesity, type 2 diabetes and neurodegenerative disorders. It thus introduces a sociological and demographic component into disease propagation and draws from certain areas in systems biology which are currently being accorded greater attention in the study of ARDs (for example, see Tacutu et al. 2010, 2011 and references therein). The mechanistic links between ARDs could largely be mediated by the phenomenon of cellular senescence (CS), which is currently suggested as one of the hallmarks of aging (Lopez-Otin et al. 2013). The role of CS in age-related pathologies is comprehensively reviewed by Ovadya and Krizhanovsky (2014), with a special focus on the role of CS in neurodegenerative diseases (Tan et al. 2014).

We believe that deep insight into the links between ARDs and aging is an essential step towards developing an efficient strategy for life- and health-span extension. The above reviews constitute an important contribution to these issues.

\section{References}

Campos-Melo D, Droppelmann CA, Volkening K, Strong MJ (2014) RNA-binding proteins as molecular links between cancer and neurodegeneration. Biogerontology. doi:10. 1007/s10522-014-9531-2

Demongeot J, Taramasco C (2014) Evolution of social networks: the example of obesity. Biogerontology. doi:10. 1007/s10522-014-9542-z

Driver JA (2014) Inverse association between cancer and neurodegenerative disease: review of the epidemiologic and biological evidence. Biogerontology. doi:10.1007/s10522014-9523-2

Fu W, Jhamandas JH (2014) Role of astrocytic glycolytic metabolism in Alzheimer's disease pathogenesis. Biogerontology. doi:10.1007/s10522-014-9525-0

Harris RA, Tindale L, Cumming RC (2014) Age-dependent metabolic dysregulation in cancer and Alzheimer's disease. Biogerontology. doi:10.1007/s10522-014-9534-z

Lopez-Otin C, Blasco MA, Partridge L, Serrano M, Kroemer G (2013) The hallmarks of aging. Cell 153:1194-1217

Ovadya Y, Krizhanovsky V (2014) Senescent cells: SASPected drivers of age-related pathologies. Biogerontology. doi:10. 1007/s10522-014-9529-9

Pellerin L, Magistretti PJ (2012) Sweet sixteen for ANLS. J Cereb Blood Flow Metab 32:1152-1166

Tacutu R, Budovsky A, Fraifeld VE (2010) The NetAge database: a compendium of networks for longevity, age-related diseases and associated processes. Biogerontology 11:513-522 
Tacutu R, Budovsky A, Yanai H, Fraifeld VE (2011) Molecular links between cellular senescence, longevity and agerelated diseases - a systems biology perspective. Aging 3:1178-1191 (Albany NY)
Tan FC, Hutchison ER, Eitan E, Mattson MP (2014) Are there roles for brain cell senescence in aging and neurodegenerative disorders? Biogerontology. doi:10.1007/s10522014-9532-1 